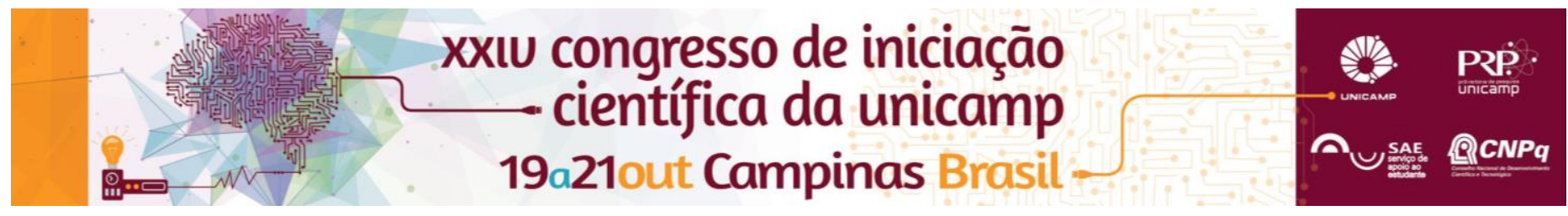

\title{
A study in wearable technology with pressure feedback for possible neurological rehabilitation treatments on hand sensory impairments.
}

\author{
Conrado F. Lago*, João H. Clerici, Mônica A. Cotta
}

\begin{abstract}
The objetive of this research is the development of wearable equipments to be used in the rehabilitation of people who suffer with somatosensory losses. The equipment substitutes the tactile response by a visual response using sensors that transduce the pressure applied on them into eletric signals. The main project consist on gloves with sensors in the fingertips; two prototypes were developed: one used for generic hand exercises and the other especifically for the pincer grasp movement rehabilitation process.
\end{abstract}

\section{Key words:}

rehabilitation, wearable sensors, somatosensory loss.

\section{Introduction}

People who suffer with somatosensory loss has the capacity of feeling pain, temperature or touch either debilitated or completely disabled in parts of the body. Stroke and other diseases and traumas that can damage the central nervous system may cause this impairment. Under these circunstances, patients can not measure their own strength causing involuntary physical effort and muscular fatigue, difficulty on writing in case of hand debilitation and loss of balance in case of foot debilitation. However, the treatment of the somatosensory loss nowadays consist mostly on physical therapy exercises, which lack a more technological approach.

This research consists on developing inexpensive technology that could aid the neuroplastic rehabilitation process. Neuroplasticity is the ability of the central nervous system to respond to intrinsic and extrinsic stimuli by reorganizing its structure, functions and connections. These brain changes are visible, for example, in the sensorimotor cortex in Braille readers ${ }^{1}$. Nowadays, this brain adaptability has a large use in rehabilitation.

In our study, our objective was to develop wearable sensors that could replace the debilitated tactile stimulus by a visual stimulus, following on original, early work by Bach-y-Rita ${ }^{2}$. In this research, we focused specifically in loss of hand sensation. For that, we used piezoelectric materials to create pressure sensors that drive the intensity of Light Emitting Diodes (LED). Our goal was to design a light and portable equipment, so that the patient could use it in domestic activities and not necessarily only during rehabilitation sections. With the constant use of the equipment, the user can adapt to the visual stimulus as a feedback of the pressure made by the fingers, and slowly gain better control of arm and hand strength, by reestablishing the neural connections between the brain cortex and the mechanoreceptors at the affected region.

\section{Results and Discussion}

Our major idea was to create a glove with pressure sensors in some of the principal points of pressure applied by the hand, transforming the corresponding force into an electric signal. This signal would turn on LED's with the intensity roughly equivalent to the applied pressure. Our main obstacle was to find pressure sensors that could be used in our project. The material needed to be small, light and flexible, so that the patient wears it with no discomfort. The feedback given by the sensors needed to be immediate and reproducible, because any variations could harm the patient development. Therefore the material also needed to present good repeatability and reproducibility, over the sensor area. We carried out tests in two materials; the one that best fitted our expectations was the Flexiforce A101, produced by Tekscan, USA. The sensor is a small circular piezoelectric material that changes resistance according to the applied pressure. Using the Flexiforce A101 sensors, two prototypes were created.

The first was created specifically for the improvement of the pincer grasp movement, which is one of the major movements of the human hand. The equipment consists on a single Flexiforce sensor placed on the tip of the thumb, and a circuit on the pulse with five LED's. The number of lit LED's depend on the range of pressure made on the sensor. The calibration of pressure ranges is made according to each patient, and can be changed upon improvement during the treatment.

The second prototype was created to be used in generic hand movements and exercises. In this case we designed a glove with a Flexiforce in each of the fingertips. On the top of each finger, there is a high luminosity LED; the light intensity varies with the pressure applied to the fingertip. Each prototype has a circuit in the pulse and are both alimented with common $3 \mathrm{~V}$ watch battery.

\section{Conclusions}

The initial tests showed that both prototypes provide immediate feedback with relative precision. Our tests show that the visual feedback signal does not vary over time and the battery lifetime seems appropriate for the use. Both prototypes do not present enough sensibility to capture light touch, but we are projecting new circuits that could solve these problems in the next versions of the equipment.

${ }^{1}$ Pascual-Leone A, Torres F. Plasticity of the sensorimotor cortex representation of the reading finger in Braille readers. Brain 1993; 116:39-52

2 Sampaio E, Maris S, Bach-y-Rita P. Brain plasticity: "visual" acuity of blind persons via the tongue. Brain Res 2001;908:204-7. 